TRANSACTIONS OF THE

AMERICAN MATHEMATICAL SOCIETY

Volume 180, June 1973

\title{
THE STRUCTURE OF DEDEKIND CARDINALS
}

\author{
BY
}

\author{
ERIK ELLENTUCK( $\left.{ }^{1}\right)$
}

ABSTRACT. Semantic criteria are given for provability in set theory without the axiom of choice of positive sentences about the Dedekind cardinals. These criteria suggest that Dedekind cardinals (as well as general cardinals) have an internal structure.

1. Introduction. Let $\omega=$ the nonnegative integers, $\Delta=$ the Dedekind cardinals, and $Z F^{0}=$ set theory with the axiom of choice replaced by the axiom of choice for sets of finite sets. For any class $A$ let $X^{n} A$ be the $n$-fold direct power of $A$. We first describe a language $L(C N)$ suitable for discussing Dedekind cardinals. The basic symbols of $L(C N)$ consist of individual variables $x_{0}, x_{1}, \cdots$; for each $n<\omega$ an almost combinatorial $f: X^{n} \omega \rightarrow \omega$, a functor $f^{\prime}$; for each $R \subseteq X^{n} \omega$, a predicate $R^{\prime}$; the binary predicate =; and the usual first order connectives and quantifiers. Terms are built up from variables and functors by composition. If $\tau_{0}, \cdots, \tau_{n-1}$ are terms and $R \subseteq X^{n} \omega$, then $\tau_{0}=\tau_{1}$ and $R^{\prime}\left(\tau_{0}, \cdots, \tau_{n-1}\right)$ are atomic formulas. The rest of the definition of formula is standard. $L(C N)$ has the obvious interpretation in $\omega$ and is interpreted in $\Delta$ by letting $f^{\prime}, R^{\prime}$ denote $f_{\Delta}, R_{\Delta}$ the canonical extensions as defined in [5]. We shall use $F$ for the satisfaction relation; thus $\Delta \vDash \mathfrak{U}$ will mean that the sentence $\mathfrak{Q}$ is true in $\Delta$. A difficulty with this notion is that in some of our applications $\Delta$ will be a class and a truth definition is not available. If this is the case we replace $\Delta \vDash \mathscr{U}$ by $\mathfrak{U}_{\Delta}$, the relativization of $\mathscr{U}$ to $\Delta$. In general we shall make no distinction between satisfaction and relativization because we are dealing with one formula at a time.

Let $(S,<)$ be the rationals with their usual ordering and let $P_{\omega}(S)$ be the set of all finite subsets of $S$. We say that a set $R \subseteq X^{k} \omega$ is cofinite if there is a finite set $a \subseteq \omega$ such that $X^{k}(\omega-a) \subseteq R$. Let $F_{k}$ be the filter of cofinite subsets of $X^{k} \omega .|a|$ will denote the cardinality of $a$. For any function $f: X^{k} \omega \rightarrow \omega$ let $f / F_{k}$ be that element in the reduced product $\omega^{x^{\bar{k}} \omega} / F_{k}$ that

Received by the editors May 2, 1972.

AMS (MOS) subject classifications (1970). Primary 02K20.

Key words and phrases. Almost combinatorial Skolem function.

(1) Prepared while the author was supported by fellowships from the Institute for Advanced Study and the New Jersey Research Council. 
corresponds to $f$. For each $A \in P_{\omega}(S)$ let $D_{A}=\left\{\left(A, f / F_{|A|+1}\right) \mid f\right.$ is an $|A|+$ 1 -ary eventually combinatorial function $\}$. Now suppose $A \subseteq B \in P_{\omega}(S)$. We are going to define a map $H_{B}^{A}: X^{|B|+1} \omega \rightarrow X^{|A|+1} \omega$. First write $A$ and $B$ in increasing order as $A=\left\{a_{0}, \cdots, a_{m-1}\right\}, a_{0}<\cdots<a_{m-1}, B=\left\{b_{0}, \cdots, b_{n-1}\right\}$, $b_{0}<\cdots<b_{n-1}$. Let $u_{A}^{B}$ be a function with domain $m$ and, for $i<m, u_{A}^{B}(i)$ is the $j$ satisfying $a_{i}=b_{j}$. Now let $x=\left(x_{0}, \cdots, x_{n}\right) \in X^{n+1} \omega$ and define $H_{B}^{A}(x)=$ $\left(y_{0}, \cdots, y_{m}\right)$ where $y_{0}=u_{A}^{B}(0)+\Sigma\left\{x_{i} \mid i \leq u_{A}^{B}(0)\right\}, y_{j}=u_{A}^{B}(j)-u_{A}^{B}(j-1)-1+$ $\Sigma\left\{x_{i} \mid u_{A}^{B}(j-1)<i \leq u_{A}^{B}(j)\right\}$ for $0<j<m$, and $y_{m}=n-u_{A}^{B}(m-1)-1+$ $\Sigma\left\{x_{i} \mid u_{A}^{B}(m-1)<i \leq n\right\}$. With $H_{B}^{A}$ we associate a mapping $\pi_{A}^{B}: D_{A} \rightarrow D_{B}$ by $\pi_{A}^{B}\left(A, f / F_{m+1}\right)=\left(B, f \circ H_{B}^{A} / F_{n+1}\right)$. Thus the family $\left\{D_{A} \mid A \in P_{\omega}(S)\right\}$ and $\left\{\pi_{A}^{B} \mid A \subseteq B \in P_{\omega}(S)\right\}$ is a directed system of algebras. Finally form the direct limit $D$ of this system. Now there is a natural way to extend each $n$-ary almost combinatorial function $f$ and $R \subseteq X^{n} \omega$ to an $f_{D}: X^{n} D \rightarrow D$ and an $R_{D} \subseteq X^{n} D$. A complete description, all computations included, can be found in [4] and [6]. Thus we can interpret $L(C N)$ in $D$. Our main results concern the relationship between the theories of $\Delta$ and $D$. As we shall see below these theories agree for a fairly comprehensive class of sentences.

Let $2 \mathcal{C}$ be a sentence of $L(C N)$. By $Z F R^{0}$ we mean $Z F^{0}$ enriched by the constants $f^{\prime}, R^{\prime}$ for each $f^{\prime}, R^{\prime}$ occurring in $\mathcal{U}$ and having additional axioms diagraming $f, R$. We do not spell out what is added, but whenever the reader sees the symbol " $Z F R{ }^{0}$ ", he is to understand that our theory diagrams the constants in any of the finitely many $\mathfrak{U} \in L(C N)$ under consideration. Let $L\left(Z F^{0}\right)$ be the language of set theory. A sentence $\mathscr{U} \in L\left(Z F^{0}\right)$ is said to be an $\omega$-consequence of $Z F^{0}$ if $\mathfrak{U}$ is true in every $\omega$-model of $Z F^{0}$, i.e., models in which the integers are standard. It is a $\beta$-consequence of $Z F^{0}$ if it is true in every $\beta$-model of $Z F^{0}$, i.e., $\omega$-models which are absolute for well-founded relations $R \subseteq X^{2} \omega$. We denote the se notions of consequence by $\vdash_{\omega}$ and $\vdash_{\beta}$ (cf. [8] for further information on $\omega$ and $\beta$-models). For the purposes of this paper we will assume that any set $s$ can be extended to a transitive model of $Z F$. Not only is this obviously true, but it is also a theorem of impredicative class-set theory.

Theorem 1. If $\mathfrak{U} \in L(C N)$ is a prenex conjunctive normal form sentence which is either universal or positive then $Z F R^{0}+\beta \mathfrak{U}_{\Delta}$ if and only if $D \vDash \mathfrak{U}$.

Let $\omega^{*}=$ the rational integers (positive, negative, zero) and let $\Delta^{*}=$ the Dedekind integers (the difference structure over $\Delta$ ). We describe a language $L^{*}(C N)$ suitable for discussing Dedekind integers. The basic symbols and formation rules of $L^{*}(C N)$ are the same as those of $L(C N)$ except for the choice of functors and predicates. $L^{*}(C N)$ contains, for each $n<\omega$ and function $f: X^{n} \omega^{*} \rightarrow \omega^{*}$, a functor $f^{\prime}$, and, for each $R \subseteq X^{n} \omega^{*}$, a predicate $R^{\prime}$. Equality is included. 
$L^{*}(C N)$ has the obvious interpretation in $\omega^{*}$ and is interpreted in $\Delta^{*}$ by letting $f^{\prime}, R^{\prime}$ denote $f_{\Delta^{*}}, R_{\Delta^{*}}$, the canonical extensions as defined in [4].

Let $D^{*}$ be the full reduced product of unary functions $g: \omega \rightarrow \omega^{*}$ modulo the cofinite subsets of $\omega . f$ and $R$ have natural extensions to $D^{*}$ (cf. [4]). Thus we can interpret $L^{*}(C N)$ in $D^{*}$. Say that a prenex conjunctive normal form sentence is disjunctive if its matrix consists of a single conjunct.

Theorem 2. If $\mathfrak{U} \in L^{*}(C N)$ is a prenex conjunctive normal form sentence which is either universal, positive, or disjunctive then $Z_{F R}{ }^{0} \vdash_{\omega} \mathfrak{R}_{\Delta^{*}}$ if and only if $D^{*} \vDash$ Q⿱ .

Actually in one direction no restriction need be placed on $\mathfrak{U}$. Namely, for any sentence $\mathfrak{U} \in L^{*}(C N)$ we have the implication $Z F R^{0} \vdash \omega^{\mathfrak{U}} \Delta^{*}$ implies $D^{*} \vDash \mathfrak{U}$. However the converse is false. Consider a sentence $B$ which says that the Boolean algebra of idempotents is atomless (this can be formulated in $L^{*}(C N)$ ). We easily see that $D^{*} \vDash B$, however $B_{\Delta^{*}}$ is not a theorem of $Z F R^{0}$ because if it were we could add the full axiom of choice to $Z F R^{0}$, force $\Delta^{*}=\omega^{*}$, and conclude that $\omega^{*} \vDash B$ which is clearly false. By formalizing this argument we can show that $Z F R^{0} \vdash_{\omega^{2}} \mathfrak{U}_{\Delta *}$ implies $\omega^{*} \vDash \mathfrak{U}$. A reasonable conjecture might be that $\omega^{*} \vDash \mathfrak{U}$ and $D^{*} \vDash \mathscr{C}$ implies $Z F R^{0} \vdash_{\omega^{2}} \mathfrak{H}_{\Delta^{*}}$. At the moment this question is open.

Theorem 2 suggests that we try to extend Theorem 1 to disjunctive sentences. Whether this can or cannot be done is also an open problem.

In a forthcoming paper we extend Theorem 1 to the general cardinals. This material is not included here because most of the work involves the development of a manageable theory of almost combinatorial series, a project which is more algebraic than set theoretic. The final results however are in the vein of Theorem 1.

We have used the word "structure" in the title of this paper for the following reason. Let $\mathfrak{U} \in L(C N)$ be a positive sentence. Suppose the prefix of $\mathscr{U}$ contains $n$ existential quantifiers and that $\mathscr{U}\left(f_{0}^{\prime}, \ldots, f_{n-1}^{\prime}\right)$ is the result of replacing these quantifiers by the $f_{i}^{\prime}$ as Skolem functors. By Theorem 1 we have $Z F R^{0} \vdash_{\beta}$ U⿱ ${ }_{\Delta}$ iff $D \vDash \mathscr{U}$ iff there exist almost combinatorial functions $f_{0}, \cdots, f_{n-1}$ such that $D \vDash \mathscr{U}\left(f_{0}^{\prime}, \cdots, f_{n-1}^{\prime}\right)$ iff $Z F R^{0} \vdash_{\beta}\left(\exists f_{0}, \cdots, f_{n-1}\right.$ almost combinatorial) $\mathcal{U}\left(f_{0}^{\prime}, \cdots, f_{n-1}^{\prime}\right)_{\Delta}$. The second and third equivalence will occur in the proof of Theorem 1. Thus if $\mathfrak{U}_{\Delta}$ is a theorem then we can actually find these Skolem functions which make $\mathfrak{U}\left(f_{0}^{\prime}, \cdots, f_{n-1}^{\prime}\right)_{\Delta}$ provable from some enriched set of axioms. This imposes a definite structure on the cardinals which $\mathfrak{U}_{\Delta}$ asserts to exist. For example if $B$ is $(\forall x)(\exists y)(x(x+1)=2 y)$ then $Z F^{0} \vdash B_{\Delta}$ by a standard set theoretic proof and hence $Z F^{0} \vdash x(x+1) / 2$ is almost combinatorial. This certainly puts structure on the value that $y$ will get in any model of $Z F^{0}$. 
2. Strong set theory. We describe a strong set theory $S T$. The language of $S T, L(S T)$, consists of the language $L\left(Z F^{0}\right)$ together with unary functors $s_{0}()$, $\bar{s}_{0}(), s_{1}(), K()$, a class symbol $K$, and a binary predicate $\prec$. In stating the axioms of $S T$ we use the defined notions $O n=$ the class of all ordinals, $P_{\omega}(A)=$ the class of all finite subsets of $A$. We use $\delta f$ and $\rho f$ for domain and range of the function $f$. All other notation is standard. The axioms of ST consist of

(1) The axioms of $Z F^{0}$ where all the symbols of $L(S T)$. may occur in the axiom of replacement.

(2) $\prec$ is a linear ordering of the universe such that if $a \in O n$ then $K_{\alpha}$ is a nonempty set and the restriction of $\prec$ to $K_{a}$ is a dense unbordered ordering.

(3) For all $\alpha, \beta \in O n$ and $x, y$ if $\alpha<\beta \wedge x \in K_{a} \wedge y \in K_{\beta}$ then $x<y$.

(4) $K=\bigcup_{a \in O_{n}} K_{a}$ and if $x, y \in K \wedge x \neq y$ then $x \cap y=\varnothing$.

(5) $s_{0}(x) \in P_{\omega}(\bigcup K), s_{1}(x) \in P_{\omega}(K), \bar{s}_{0}(x)=\left\{z \mid(\exists y) y \in s_{0}(x) \wedge y \in z \in K\right\}$, and $\bar{s}_{0}(x) \subseteq s_{1}(x)$.

(6) If $x \in y \in K$ then $s_{0}(x)=\{x\}, s_{1}(x)=\{y\}, s_{0}(y)=\varnothing, s_{1}(y)=\{y\}$. If $\alpha \in O n$ and $j<2$ then $s_{j}(\alpha)=s_{j}\left(K_{\alpha}\right)=\varnothing$.

(7) If $y$ is finite and $j<2$ then $s_{j}(y)=\bigcup\left\{s_{j}(x) \mid x \in y\right\}$.

(8) If $y$ is finite then the restriction of $\prec$ to the class $\left\{x \mid s_{0}(x) \cup s_{1}(x) \subseteq y\right\}$ is a well ordering.

(9) Schema: if $\phi\left(y_{1} x_{0}, \cdots, x_{n-1}\right)$ is a formula with free variables indicated and $j<2$ then the closure of $(\exists ! z) \phi\left(z, x_{0}, \cdots, x_{n-1}\right) \wedge \phi\left(y, x_{0}, \cdots, x_{n-1}\right) \rightarrow$ $s_{j}(y) \subseteq \bigcup_{i<n} s_{j}\left(x_{i}\right)$ is an axiom.

In order to state the rest of our axioms we need

Definition. aut $(\pi)$ iff $\pi$ is a finite function $\wedge \delta \pi \cup \rho \pi \subseteq K \wedge \pi$ is $\prec$ order preserving $\Lambda$ if $x \in \delta \pi \cap K_{a}$ then $\pi(x) \in K_{a}$.

Definition. $\operatorname{acp}(\pi, x)$ iff aut $(\pi) \wedge s_{1}(x) \subseteq \delta \pi \wedge \bar{s}_{0}(x)$ is left pointwise fixed by $\pi$.

(10) The action of $\pi$ satisfying aut $(\pi)$ is extended to $x$ satisfying $\operatorname{acp}(\pi, x)$.

(11) If $x \in y \in K$ and aut $(\pi)$ then $\operatorname{acp}(\pi, y)$ iff $y \in \delta \pi$ and in this case $\pi(y)$ has its natural value, $\operatorname{acp}(\pi, x)$ iff $y \in \delta \pi \wedge \pi(y)=y$ and in this case $\pi(x)=x$. If $\alpha \in O n$ and aut $(\pi)$ then $\operatorname{acp}(\pi, \alpha) \wedge \operatorname{acp}\left(\pi, K_{\alpha}\right) \wedge \pi(\alpha)=\alpha \wedge \pi\left(K_{\alpha}\right)$ $=K_{\alpha}$.

(12). If $\operatorname{acp}(\pi, y)$ and $(\forall x \in y) \operatorname{acp}(\pi, x)$ then $\pi(y)=\{\pi(x) \mid x \in y\}$.

(13) If $\operatorname{acp}(\pi, x) \wedge$ aut $\left(\pi^{\prime}\right) \wedge \rho \pi \subseteq \delta \pi^{\prime} \wedge \pi^{\prime}$ leaves $\bar{s}_{0}(x)$ pointwise fixed then $\operatorname{acp}\left(\pi^{\prime} \pi, x\right) \wedge \operatorname{acp}\left(\pi^{\prime}, \pi(x)\right) \wedge\left(\pi^{\prime} \pi\right)(x)=\pi^{\prime}(\pi(x))$.

(14) If $\operatorname{acp}(\pi, x)$ and $s_{1}(x)$ is left pointwise fixed by $\pi$ then $\pi(x)=x$.

(15) Schema: if $\phi\left(x_{0}, \ldots, x_{n-1}\right)$ is a formula with free variables indicated then the closure of $\bigwedge_{i<n}$ acp $\left(\pi, x_{i}\right) \wedge \phi\left(x_{0}, \cdots, x_{n-1}\right) \rightarrow \phi\left(\pi\left(x_{0}\right), \cdots, \pi\left(x_{n-1}\right)\right)$ is an axiom. 
This concludes our list of axioms. As the reader may have guessed, we are dealing with a Cohen extension where we have adjoined sets of ordinals, and sets of these sets. This accounts for the functors $s_{0}$ and $s_{1}$ giving two kinds of support to elements of our universe. In the final section of this paper we prove

Theorem 3. If $M$ is a countable transitive model of $Z F$ for which the re is a definable well ordering then $M$ has a transitive extension $N$ which is a model of $S T$, having the same ordinals and well-orderable cardinals as $M$.

We introduce the following notation. For each $n<\omega$, an $n$-tuple $x=$ $\left(x_{0}, \cdots, x_{n-1}\right)$ is a function with $\delta x=n$. For any class $A$ we let $\mathrm{X}^{n} A=$ the class of all $n$-tuples $x$ with $\rho x \subseteq A . \quad V$ is the class of all sets and $Q$ is the class of all finite sets. We let $|x|$ be the cardinal number of $x$ and write $x \sim y$ for $x$ is equipollent with $y$. More generally $p: x \sim y$ will mean that $p$ is a oneone function mapping $x$ onto $y$. When referring to elements of $X^{n} V$ we understand this notation as being used componentwise. Thus if $x, y \in X^{n} V$ then $|x|_{i}=\left|x_{i}\right|$ for $i<n$, and $x \subseteq y$ if $x_{i} \subseteq y_{i}$ for $i<n$. If $\alpha \in O n$ and $a \in Q$ we let $K_{a}(a)$ be the finite sequence of open intervals with respect to $\prec$ whose union is $K_{a}-a$ and which is determined by $K_{a} \cap a$. The order of these intervals in $K_{a}(a)$ agrees with the $\prec$ ordering. If $s \in P_{\omega}(O n)$ then $K_{s}(a)$ is the concatenation of the $K_{\alpha}(a)$ for $a \in s$ where again the order of the intervals in $K_{s}(a)$ agrees with the $\prec$ ordering. We leave unexplained all the notation connected with combinatorial operators and frames as applied to set theory. The reader will find a more than adequate explanation in [5]. Throughout the rest of this section we work in the theory $S T$.

Lemma 1. If $a \in O n$ then $K_{\alpha}$ is an infinite Dedekind set.

Proof. By (2), $K_{\alpha}$ is infinite. Assume that $f: \omega \rightarrow K_{a}$. If $x \in \rho f$ then $x=$ $f(n)$ for some $n<\omega$. Then $\{x\}=s_{1}(x) \subseteq s_{1}(f) \cup s_{1}(n)=s_{1}(f) \in P_{\omega}(K)$ by (6), (9) and (5). Hence $\rho f \subseteq s_{1}(f)$ is finite. But this implies that $K_{a}$ is Dedekind. Q.E.D.

Lemma 2. Let $F \subseteq X^{m+n} Q$ be a frame, $y \in X^{m} V, s \in P_{\omega}(O n)$, and $a \in Q$ such that $K_{s}(a) \in X^{n} V$. If $\left(y, K_{s}(a)\right.$ ) is attainable from $F$ (in symbols $\left.\left(y, K_{s}(a)\right) \in \mathbb{P}(F)\right)$ then there exists $a b \in X^{n} Q, b \subseteq K_{s}(a)$, such that for all $d \in X^{n} Q, b \subseteq d \subseteq K_{s}(a)$ implies that $(y, d) \in \mathbb{P}(F)$.

Proof. Let $z=K_{s}(a)$ and assume that $(y, z) \in \mathbb{P}(F)$. Let $b=\left(b_{0}, \cdots, b_{n-1}\right)$ where $b_{i}=z_{i} \cap\left(s_{1}(F) \cup s_{1}(y)\right)$. For any $d \in X^{n} Q$ with $b \subseteq d \subseteq z$ let $e$ be the last $n$ components of $C_{F}(y, d)$. Consider any $j<n$ and $x \in e_{j}$. Now $e \subseteq z$ and 
hence $\{x\}=s_{1}(x) \subseteq s_{1}\left(e_{j}\right) \subseteq s_{1}(e) \subseteq s_{1}(F) \cup s_{1}(y) \cup s_{1}(d)$ by (6), (7) and (9). Now $x \in z_{j}$ and hence $x \in b_{j}$ or $x \in s_{1}(d)$. But $s_{1}(d)=\bigcup_{i<n} s_{1}\left(d_{i}\right)$ by (7) so $x \in d_{j}$. Thus $d=e$ and we conclude that $C_{F}(y, d)=(y, d) \in \mathbb{Q}(F)$. Q.E.D.

Corollary 1. Let $R \subseteq X^{m+n} \omega, y \in X^{m} \Delta, s \in P_{\omega}(O n)$, and $a \in Q$ such that $K_{s}(a) \in X^{n} V$. If $\left(y,\left|K_{s}(a)\right|\right) \in R_{\Delta}$ then $\left\{z \in X^{n} \omega \mid(y, z) \in R_{\Delta}\right\}$ is a cofinite set.

Corollary 2. Let $R_{i} \subseteq X^{m+n} \omega$ for $i<k$ and $y \in X^{m} \Delta$. If $\left(\forall z \in X^{n} \Delta\right)$ $\bigvee_{i<k}(y, z) \in R_{i \Delta}$, then there is a $j<k$ such that $\left\{z \in X^{n} \omega \mid(y, z), \in R_{j \Delta}\right\}$ is a cofinite set.

Corollary 3. Let $R_{i} \subseteq X^{m+n} \omega^{*}$ for $i<k$ and $y \in X^{m} \Delta^{*}$. If $\left(\forall z \in X^{n} \Delta^{*}\right)$ $\bigvee_{i<k}(y, z) \in R_{i \Delta^{*}}$ then there is a $j<k$ sucb that $\left(\forall z \in X^{n} \omega^{*}\right)(y, z) \in R_{j \Delta^{*}}$.

Corollary 4. The Boolean algebra of idempotent elements in $\Delta^{*}$ is atomless (cf. [7]).

Proof. Define a function $f$ on $\mathrm{X}^{2} \omega^{*}$ by $f(x, 2 y)=x$ and $f(x, 2 y+1)=0$. The following formulas about $f$ are valid in $\omega^{*}$. (i) $x^{2}=x \rightarrow f(x, y)^{2}=f(x, y)$, (ii) $x^{2}=x \rightarrow f(x, y) \cdot x=f(x, y)$, (iii) $f(x, 2 y)=0 \rightarrow x=0$, and (iv) $f(x, 2 y+1)$ $=x \rightarrow x=0$. These are Horn sentences and hence by Theorem 2 of [4] are valid in $\Delta^{*}$. Now let $u \in \Delta^{*}$ be an idempotent which differs from 0 . For any $y \in \Delta^{*}$, (i) implies that $f_{\Delta^{*}}(u, y)$ is an idempotent and (ii) implies that $0 \leq f_{\Delta^{*}}(u, y) \leq u$ in the Boolean algebra ordering. Now suppose that $\left(\forall y \in \Delta^{*}\right) f_{\Delta^{*}}(u, y)=0 \vee f_{\Delta^{*}}(u, y)=u$. Corollary 3 then implies that either $\left(\forall y \in \omega^{*}\right) f_{\Delta^{*}}(u, y)=0$ or $\left(\forall y \in \omega^{*}\right) f_{\Delta^{*}}(u, y)=u$. But (iii) and (iv) in both cases give $u=0$, a contradiction. Thus there is a $y \in \Delta^{*}$ such that $0<f_{\Delta^{*}}(u, y)<u$. Q.E.D.

Corollary 5. For any sentence $\mathfrak{U} \in L^{*}(C N), \mathfrak{U}_{\Delta^{*}}$ if and only if $D^{*} \vDash \mathfrak{U}$.

Proof. In [10] it is shown that the first order theory of arithmetic isolic integers is the same as that of $D^{*}$. The only tools used in the proof are the isolic versions of Corollary 4 above and Theorem 2 of [4]. Thus that result immediately transfers to $\Delta^{*}$. Q.E.D.

Lemma 3. Assume that $y \in X^{n} V, F \subseteq X^{n} Q$ is a frame, $y \in \mathbb{P}(F), s_{1}(y) \cup$ $s_{1}(F) \subseteq \bigcup_{a \in s} K_{a}$ where $s \in P_{\omega}(O n)$, and $|y| \in X^{n} \Delta$. Then there exist $b \in Q$, $m<\omega$ with $K_{s}(b) \in X^{m} V, y^{\prime} \subseteq y$ and m-ary combinatorial operators $\phi_{i}$ for $i<m$ such that $y^{\prime} \in \mathbb{Q}(F)$ and each $y_{i}^{\prime}$ bas a representation of the form $y_{i}^{\prime} \sim \phi_{i}\left(K_{s}(b)\right)$.

Proof. Let $a=s_{0}(y) \cup s_{0}(F), b=s_{1}(y) \cup s_{1}(F)$, and $y_{i}^{\prime}=\left\{x \in y_{i} \mid s_{1}(x) \subseteq\right.$ 
$\left.\bigcup_{a \in s} K_{a} \wedge s_{0}(x) \subseteq a\right\}$. First we show that $y^{\prime} \in\left(\mathcal{P}(F)\right.$. Let $d \in X^{n} Q, d \subseteq y^{\prime}$ and $C_{F}(d)=e$. Consider any $i<n, j<2$ and $x \in e_{i}$. Then $s_{j}(x) \subseteq s_{j}\left(e_{i}\right) \subseteq s_{j}(e) \subseteq$ $s_{j}(d) \cup s_{j}(F)$ by (7) and (9). But $s_{j}(d)=\bigcup_{i<n} \bigcup\left\{s_{j}(x) \mid x \in d_{i}\right\}$ by (7) so $s_{0}(x) \subseteq a$ and $s_{1}(x) \subseteq \bigcup_{a \in s} K_{a}$. Thus $x \in y_{i}^{\prime}$ and hence $C_{F}(d) \subseteq y^{\prime}$. But this means that $y^{\prime} \in \mathbb{Q}(F)$. Let $H=\left\{\pi \mid\right.$ aut $(\pi) \wedge b \subseteq \delta \pi \subseteq \bigcup_{a \in s} K_{a} \wedge b$ is left pointwise fixed by $\pi\}$. Now $\bar{s}_{0}\left(y_{i}^{\prime}\right) \subseteq s_{1}\left(y_{i}^{\prime}\right) \subseteq s_{1}(y) \cup s_{1}(a) \subseteq s_{1}(y) \cup s_{1}(F)=b$ by (5), (7) and (9). Hence if $\pi \in H$ then acp $\left(\pi, y_{i}^{\prime}\right)$ and $\pi\left(y_{i}^{\prime}\right)=y_{i}^{\prime}$ by (14). If $x \in y_{i}^{\prime}$, $\pi \in H$ and $s_{1}(x) \subseteq \delta \pi$, then $\operatorname{acp}(\pi, x)$ because $s_{0}(x) \subseteq a$, i.e., $\bar{s}_{0}(x) \subseteq b$. Then $\pi(x) \in \pi\left(y_{i}^{\prime}\right)$ by (15). For any $x, x^{\prime} \in y_{i}^{\prime}$ let $x \approx x^{\prime}$ if there is a $\pi \in \epsilon^{\prime} H$ with $\operatorname{acp}(\pi, x)$ such that $\pi(x)=x^{\prime}$. Using (13) and (14) we easily show that $\approx$ is an equivalence relation. Let $[x]$ be the equivalence class of $x$ and let $A=\left\{[x] \mid x \in y_{i}^{\prime}\right\} . A$ is a partition of $y_{i}^{\prime}$. If $u \in A$ and $x, x^{\prime} \in u$ then $x^{\prime}=\pi(x)$ for some $\pi \in H$ with $s_{1}(x) \subseteq \delta \pi$. But $s_{1}(\pi(x))=\pi\left(s_{1}(x)\right)=\left\{\pi(z) \mid z \in s_{1}(x)\right\}$ by (15) and (7). Hence if $s_{1}(x)=s_{1}\left(x^{\prime}\right)$ then $\pi$ is the identity on $s_{1}(x)$ so $x=x^{\prime}$ by (14). Let $K_{s}(b) \in X^{m} V$. For $x \in u$ define $f(x)=z \in X^{m} Q$ by $z_{j}=s_{1}(x) \cap$ $K_{s}(b)_{j}$. If $x, x^{\prime} \in u$ and $f(x)=f\left(x^{\prime}\right)$ then $s_{1}(x)-b=s_{1}\left(x^{\prime}\right)-b$ so $x=x^{\prime}$. Thus $f$ is one-one on $u$. Also note that for $x, x^{\prime} \in u$ we have $|f(x)|=\left|f\left(x^{\prime}\right)\right|$ componentwise. For any set $S$ and $k<\omega$ let $(S / k)=$ the set of all $k$ element subsets of $S$. If $S \in X^{m} V$ and $k \in X^{m} \omega$ let $(S / k)=\left(S_{0} / k_{0}\right) \times \cdots \times\left(S_{m-1} / k_{m-1}\right)$. Thus if $x \in u$ and $|f(x)|=k$ then $\{f(x) \mid x \in u\} \subseteq\left(K_{s}(b) / k\right)$. We claim that the map is onto. Let $z \in\left(K_{s}(b) / k\right)$ and $x \in u$. Then there is a $\pi \in H$ such that $\pi\left(s_{1}(x)\right)=z$. But $\pi(x) \in u$, moreover $s_{1}(\pi(x))=\pi\left(s_{1}(x)\right)=\dot{z}$. For each $u \in A$ construct a map $f_{u}$ as above. For each $k \in X^{m} \omega$ we claim that there are at most finitely many $u \in A$ such that $u$ is mapped onto $\left(K_{s}(b) / k\right)$. For otherwise we could find an infinite set $v \subseteq y_{i}^{\prime}$ and a $k \in X^{m} \omega$ such that distinct elements of $v$ belong to distinct equivalence classes, but each gets mapped to the same element of $\left(K_{s}(b) / k\right)$. But then by (8) the restriction of $\prec$ to $v$ is a well ordering which contradicts the fact that $y_{i}$ is Dedekind. Let $c(k)=\left\{u \leq A \mid f_{u}: u \sim\right.$ $\left.\left(K_{s}(b) / k\right)\right\}$. Since $c(k)$ is finite $\prec$ gives it a standard well ordering and we may construct a map $f_{k}: \bigcup_{c}(k) \sim|c(k)| \times\left(K_{s}(b) / k\right)$. If we take the $|c(k)|$ as Stirling coefficients of an $m$-ary combinatorial function and we let $\phi_{i}$ be the associated normal combinatorial operator then we can piece the various $f_{k}$ together and get $y_{i}^{\prime} \sim \phi_{i}\left(K_{s}(b)\right)$. Q.E.D.

Consider any sentence $\mathfrak{U} \in L(C N)$ which has the form $\left(\forall x_{0}\right) \cdots\left(\forall x_{n-1}\right) \mathfrak{B}$ where $B$ is quantifier free and is written in conjuctive normal form. With $\mathscr{X}$ we associate a sentence cond (U) $\in L(C N)$ which means when interpreted in $\omega$ that IC holds and whenever we specify definite integer values to replace some, but not all, of the variables $x_{i}$ in $B$ then $B$ has a Horn reduct $B^{\prime}$ such that, subject to 
the replacement made above, the set of integers that satisfy $B^{\prime}$ is a cofinite set. The reader can find a precise definition of cond in [3]. For readers familiar with the theory of isols, cond is simply the Nerode condition for the truth of universal sentences in the isols (cf. [9]).

Lemma 4. Let $R_{i} \subseteq X^{n} \omega$ for $i<t$ and let $Q_{i}$ be a quantifier for $i<n$. Let $\mathscr{U}$ be the sentence $\left(Q_{0} x_{0}\right) \ldots\left(Q_{n-1} x_{n-1}\right) \mathbb{B}$ where $B$ is $R_{0}^{\prime}\left(x_{0}, \cdots, x_{n-1}\right) \vee \cdots$ $\vee R_{t-1}^{\prime}\left(x_{0}, \cdots, x_{n-1}\right)$. Let $\tilde{\mathscr{U}}$ be the result of omitting the existential quantifiers from the prefix of $\mathfrak{Q}$ and replacing existentially quantified variables of $\mathfrak{B}$ by suitably placed Skolem functors $f_{i}^{\prime}$. Then $\mathfrak{A}_{\Delta}$ implies that there exist almost combinatorial functions $f_{i}$ such that $\omega \vDash$ cond $(\widetilde{\mathscr{U}})$ where we un derstand that $f_{i}^{\prime}$ denotes $f_{i}$

Proof. Let $u=\left\{i<n \mid Q_{i}\right.$ is universal $\}$ and $e=\left\{i<n \mid Q_{i}\right.$ is existential $\}$. Let $\sigma \subseteq u$ and $q: \sigma \rightarrow \omega$. We construct a sequence of sets $y \in X^{n} V$ as follows. First we require that $|y| \in X^{n} \Delta$ and that $\left|\left(y_{0}, \ldots, y_{j}\right)\right|$ satisfies the partial formulas $\left(Q_{j+1^{x}{ }_{j+1}}\right) \cdots\left(Q_{n-1^{x} n-1}\right) B$ in $\Delta$ for each $j<n$. If $j \in \sigma$ then $y_{j}$ is a finite set of $q(j)$ integers, and if $j \in u-\sigma$ then $y_{j}=K_{a}$ where $\alpha$ is the least ordinal greater than each element of $\left\{\xi \mid(\exists i<j)\left(\exists x \in y_{i}\right) s_{1}(x) \cap K_{\xi} \neq \varnothing\right\}$. The axiom of replacement gives us such an $\alpha$. $|y|$ satisfies some disjunct of $B$, say $|y| \in R_{\Delta}$. Hence $y \in \mathbb{Q}(F)$ for some $R$-frame $F$. Let $s \in P_{\omega}(O n)$ be the least set such that $s_{1}(y) \cup s_{1}(F) \subseteq \bigcup_{a \in s} K_{a}$ and if some $y_{r}=K_{a}$ then $a \in s$. Apply Lemma 3 to get $y^{\prime} \subseteq y, y^{\prime} \in \mathbb{Q}(F), b \in Q, K_{s}(b) \in X^{m} V$, and $m$-ary combinatorial operators $\phi_{i}$ such that $y_{i}^{\prime} \sim \phi_{i}\left(K_{s}(b)\right)$ for $i<n$. It follows from the definition of $y_{i}^{\prime}$ that (i) if $i \in \sigma$ then $y_{i}^{\prime}=y_{i}$ and we may take $\phi_{i}(x)$ to have the constant value $y_{i}$, (ii) if $i \epsilon u-\sigma, y_{i}=K_{a}$ then $y_{i}^{\prime}=K_{a} \sim \bigcup_{j} K_{a}(b){ }_{j} \cup\left(K_{a} \cap b\right)$. If $b(i), k(i)$ are the indices of the first, last component of $K_{a}(b)$ in $K_{s}(b)$ then we may take $\phi_{i}(x)=x_{b(i)}+\cdots+x_{k(i)}+\left|K_{a} \cap b\right|$, and (iii) if $i \in e$ and $j$ is the least element such that $i<j \in u-\sigma$ then $\phi_{i}(x)$ depends only on its first $b(j)$ arguments. Let $f_{i}$ be the combinatorial function induced by $\phi_{i}$ and let $f_{\Delta}(x)=\left(f_{0 \Delta}(x), \cdots\right.$, $\left.f_{(n-1) \Delta}(x)\right)$. This gives $f_{\Delta}\left(\left|K_{s}(b)\right|\right) \in R_{\Delta}$. Define $S=\left\{x \in X^{m} \omega \mid f(x) \in R\right\}$. Then by [5], $\left|K_{s}(b)\right| \in S_{\Delta}$ so by Corollary 1 (without parameters) there is an $\bar{a}=$ $(a, \cdots, a) \in X^{m} \omega$ such that if $\bar{a} \leq x \in X^{m} \omega$ then $x \in S$ and hence $f(x) \in R$. Let $E=\{x \mid x: u-\sigma \rightarrow \omega\}$. For each $x \in E$ let $p(x)=z \in X^{m} \omega$ be defined as follows. If $j \in u-\sigma$ then $z_{b(j)}=x_{j}+a, z_{j}=a$ in all other cases. Let $g_{j}(x)=$ $f_{j}(p(x))$ for $x \in E$ and $j<n$. Then the $g_{j}$ are combinatorial functions and (i) if $j \in \sigma$ then $g_{j}(x)$ has the constant value $\left|y_{j}\right|$, (ii) if $j \in u-\sigma$ then $g_{j}(x)=x_{j}+\mathrm{a}$ constant integer, and (iii) if $j \in e$ then $g_{j}(x)$ depends only on the restriction of $x$ to $\{i \in u-\sigma \mid i<j\}$. We also see that $g(x) \in R$ for all $x \in E$ where 
$g(x)=\left(g_{0}(x), \cdots, g_{n-1}(x)\right)$. Thus from $g$ we can construct eventually combinatorial Skolem functions $b_{j}$ for $j \in e$ such that, if $\widetilde{R}$ results from $R^{\prime}\left(x_{0}, \cdots, x_{n-1}\right)$ by replacing the variables $x_{j}$ for $j \in e$ by $b_{j}^{\prime}$ where the arguments of $b_{j}^{\prime}$ are the $x_{i}$ 's with $i \in u-\sigma \wedge i<j$, then $\widetilde{R}$ is true in $\omega$ when sufficiently large values are assigned to the $x_{j}, j \in u-\sigma$, and $q(j)$ is assigned to $x_{j}, j \in \sigma$. Now recall that this whole construction was done for a specific $q$. As we vary $q$ the $y \in$ $\mathrm{X}^{n} V$ may satisfy a different disjunct of $B$. In any event we can piece together almost combinatorial Skolem functions $b_{i}$ for $i \in e$ such that if $\tilde{B}$ is the result of replacing $x_{i}$, $i \in e$, by $b_{i}^{\prime}$ then $\widetilde{B}$ has the following property. For every $\sigma \subseteq u$ and $q: \sigma \rightarrow \omega$ there is a disjunct $\widetilde{R}$ of $\widetilde{B}$ which holds in $\omega$ when large enough values are assigned to the $x_{j}, j \in u-\sigma$, and $q(j)$ is assigned to $x_{j}, j \in \sigma$. But

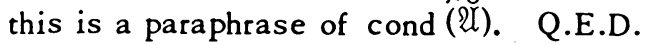

Proof of Theorem 1. Let $\mathfrak{A} \in L(C N)$ be a sentence such that $D \vDash \mathfrak{U}$. It is an easy matter to check that if $\mathscr{U}$ is universal then $\omega \vDash$ cond ( $\mathscr{U})$. Now the latter is a first order arithmetical condition and hence by the $\omega$-rule $Z F R^{0} \vdash_{\omega}$ cond( $(\mathscr{T}) \omega$. But then by Theorem 7 of [3], ZFR ${ }^{0} \vdash_{\omega} \mathfrak{A}_{\Delta}$. Note that (i) this implication is slightly stronger than the claim of Theorem 1, i.e., the $\omega$-rule and not the $\beta$-rule is required, and (ii) the definition of cond used in this paper is slightly stronger than that of [3]. If $\mathfrak{A}$ is positive, then by Lemma 4 of [6] there exist almost combinatorial Skolem functions such that $\omega$ F cond $(\tilde{\mathscr{U}})$. Now this is a $\Sigma_{1}^{1}$ second order arithmetical condition and hence by $[8]$ is absolute for $\beta$-models. Then $Z F R^{0} \vdash_{\beta}$ there exist almost combinatorial Skolem functions such that cond $(\widetilde{\mathscr{U}})_{\omega}$. By Theorem 7 of [3] and predicate logic, $Z F R^{0} \vdash_{\beta} \mathfrak{H}_{\Delta}$. Conversely suppose II $\epsilon$ $L(C N)$ and $Z F R^{0} r_{\beta} \mathfrak{U}_{\Delta}$. Start with a countable transitive model $M$ of $Z F^{0}$ which contains the functions $f$ and relations $R$ denoted by constants occurring in $\mathfrak{U}$. By looking at the submodel of sets in $M$ constructible from $f$ and $R$ we may also assume that $M$ meets the hypothesis of Theorem 3 so $N \vDash \mathscr{A}_{\Delta^{\cdot}}$. If $\mathscr{U}$ is universal then by Theorem 7 of [3] we have $N$ F cond (U) $\omega$. Since $N$ is an $\omega$-model and hence absolute for first order arithmetic we get $\omega \vDash$ cond (U). By an easy argument this gives $D \vDash \mathfrak{U}$. If $\mathscr{U}$ is positive then by Lemma $4, N \vDash$ there exist almost combinatorial Skolem functions such that cond $(\widetilde{\mathscr{U}})_{\omega}$. By absoluteness we have $\omega \vDash$ cond $(\widetilde{\mathfrak{U}})$ and then $D \vDash \widetilde{\mathscr{U}}$ and finally $D \vDash \mathscr{U}$. Q.E.D.

Proof of Theorem 2. Let $\mathfrak{A} \in L^{*}(C N)$ be a sentence which is either universal, positive or disjunctive. Then $D^{*} F \mathscr{U}$ iff there is a Horn reduct $\mathfrak{U}^{\prime}$ of $\mathscr{U}$ such that $\omega^{*} \vDash \mathfrak{U}^{\prime}$ by Corollary 3 of [3]. Corollary 5 then implies that $D^{*} \vDash \mathfrak{A}$ iff $\omega^{*} \vDash \mathfrak{U}^{\prime}$ iff $Z F R^{0} \vdash_{\omega^{*}} \mathfrak{X}_{\omega^{*}}^{\prime}$ iff $Z F R^{0} \vdash_{\omega^{2}} \mathfrak{I}_{\Delta^{*}}^{\prime}$ iff $Z F R^{0} \vdash_{\omega^{2}} \mathfrak{U}_{\Delta^{*}}$ Q.E.D.

3. The consistency of $S T$. Let $M$ be a countable transitive model of $Z F$ including the axiom of choice. Assume that the following classes are definable 
in $M$. A well ordering $W$ of $M$, functors $\left.\theta_{(}\right)$and $K_{(}$) where for each $a \in O n^{(M)}$ (superscript denotes relativization), $\theta_{a}$ is the $\alpha_{\text {th }}$ regular cardinal and $K_{a}$ is a set of cardinality $\theta_{\alpha}$, and the $K_{\alpha}$ 's are pairwise disjoint, the class $K=$ $\bigcup\left\{K_{a} \mid \alpha \in O n^{(M)}\right\}$, and a linear ordering $\prec$ of $K$ such that the restriction of $\prec$ to $K_{a}$, i.e., $\prec \mid K_{\alpha}$ is dense unbordered for each $\alpha \in O n^{(M)}$, and if $x \in K_{a} \wedge y \in$ $K_{\beta} \wedge a<\beta$ then $x<y$.

We define a ramified language $L(R A)$ in $M$. The symbols of $L(R A)$ are individual variables $x_{0}, x_{1}, \ldots$, binary predicates $\varepsilon$,, connectives $\Lambda, \sim$, quantifiers $\forall_{a}$ for each $\alpha \in O n^{(M)}$, and set builders $g_{\alpha}$ for each $\alpha \in O n^{(M)}$. We also assume that $L(R A)$ contains the following categories of individual constants (which can be uniquely read in $M$ ): an $m$ for each $m \in M$, for each $\alpha \in O n^{(M)}$ constants $K \mid \alpha^{\sim},\langle| \alpha^{\sim}, K_{\alpha}^{\sim}, \prec_{a}^{\sim}$, for each $k \in K_{\alpha}$ a.constant $k^{\sim}$, and for each $\beta<\theta_{a}$ a constant $(k, \beta)^{\swarrow}$. We simultaneously recursively define the terms and formulas of $L(R A)$ as well as two functions $\lambda$ and occ. Each individual variable $x$ is a term, $\lambda(x)=0$, and $\operatorname{occ}(x)=\varnothing$. $m$ is a term for each $m \in M$, $\lambda(\mathrm{m})$ is the set theoretic rank of $m$, and $\operatorname{occ}(\mathrm{m})=\varnothing$. If $k \in K_{\alpha}$ and $\beta<\theta_{a}$ then $(k, \beta)^{\smile}$ is a term, $\lambda(k, \beta)^{\swarrow}=\theta_{a}$, and occ $(k, \beta)^{\swarrow}=\{(k, \beta)\}$. $k^{\llcorner}$is also a term, $\lambda\left(k^{\swarrow}\right)=\theta_{a}+1$, and $\operatorname{occ}\left(k^{\sim}\right)=\{k\}$. If $\alpha \in O n^{(M)}$ then $K_{\alpha}^{\sim}$ is a term, $\lambda\left(K_{a}^{\swarrow}\right)=$ $\theta_{\alpha}+2$, and $\operatorname{occ}\left(K_{\alpha}^{\sim}\right)=\varnothing . \prec_{a}^{\sim}$ is also a term, $\lambda\left(\prec_{\alpha}^{\sim}\right)=\theta_{\alpha}+4$, and $\operatorname{occ}\left(\prec_{\alpha}^{\sim}\right)=\varnothing$. Finally if $\alpha \in O n^{(M)}$ then $K \mid \alpha^{2}$ is a term, $\lambda\left(K \mid \alpha^{2}\right)=\sup \left\{\theta_{\beta}+4 \mid \beta<\alpha\right\}$, and $\operatorname{occ}\left(K \mid \alpha^{2}\right)=\varnothing$. \langle|$\alpha^{\sim}$ is also a term, $\lambda\left(\prec \mid \alpha^{2}\right)=\sup \left\{\theta_{\beta}+\sigma \mid \beta<\alpha\right\}$, and $\operatorname{occ}\left(\langle| \alpha^{2}\right)=\varnothing$. If $u, v$ are terms and $\phi$ is $u \varepsilon v$ or $u \equiv v$ then $\phi$ is a formula, $\lambda(\phi)=1+\max \{\lambda(u), \lambda(v)\}$, and $\operatorname{occ}(\phi)=\operatorname{occ}(u) \cup \operatorname{occ}(v)$. If $\phi, \psi$ are formulas then so is $\phi \wedge \psi, \lambda(\phi \wedge \psi)=\max \{\lambda(\phi), \lambda(\psi)\}$, and $\operatorname{occ}(\phi \wedge \psi)=\operatorname{occ}(\phi) \cup$ $\operatorname{occ}(\psi) \cdot \sim \phi$ is also a formula, $\lambda(\sim \phi)=\lambda(\phi)$, and $\operatorname{occ}(\sim \phi)=\operatorname{occ}(\phi)$. If $\phi$ is a formula, $x$ is a variable, and $\alpha \in O n^{(M)}$, then $\left(\forall_{a} x\right) \phi$ is a formula, $\lambda\left(\forall_{a} x\right) \phi=$ $\max \{\alpha, \lambda(\phi)\}$, and $\operatorname{occ}\left(\forall \forall_{\alpha}\right) \phi=\operatorname{occ}(\phi)$. Finally if $\phi$ is a formula, $x$ is a variable, $\alpha \in O n^{(M)}$, and $\lambda(\phi) \leq \alpha$ then $\left(g_{\alpha} \bar{x}\right) \phi$ is a term, $\lambda\left(g_{a} x\right) \phi=\alpha$, and $\operatorname{occ}\left(g_{a} x\right) \phi=\operatorname{occ}(\phi)$. This completes our definition of $L(R A)$. A constant term is one in which no variable appears freely, and a sentence is a formula in which no variable appears freely. Let $T(R A)$ be the class of constant terms and $S(R A)$ the class of sentences.

A condition $p$ is a set (in $M$ ) of 5 -tuples ( $\alpha, k, \beta, \gamma, i)$ such that (i) $\alpha \in O_{n}^{(M)}, k \in K_{a},\{\beta, \gamma\} \subseteq \theta_{a}$, and $i<2$, (ii) $p$ is consistent in the sense that $(\alpha, k, \beta, \gamma, 0)$ and $(\alpha, k, \beta, \gamma, 1)$ cannot both belong to $p$, and (iii) if $p^{\alpha}$ is the set of all 5-tuples in $p$ that begin with $\alpha$ then $\left|\bigcup\left\{p^{\beta} \mid \beta \leq a\right\}\right|^{(M)}<\theta_{a}$. Let $C$ be the class of all conditions. We partially order $C$ by inclusion and understand $p \subset q$ to mean that $q$ contains more information than $p$. When we speak 
of a class of $M$ we mean a subset of $M$ (not necessarily in $M$ ), which can be defined in $M$ with the aid of individuals of $M$ as parameters. Since $K_{(}$) and $\theta_{(\text {) }}$ are functors over $M$ it is clear that $T(R A), S(R A)$ and $C$ are classes of $M$. A class $D \subseteq C$ is dense open if (i) $p \in C$ implies that there is a $q \in D$ with $p \subseteq q$, and (ii) $p \in D$ and $p \subseteq q \in C$ implies that $q \in D$. A subset $G \subseteq C$ is called $C$ generic over $M$ if (i) $p \in C$ and $p \subseteq q \in G$ implies that $p \in G$, (ii) $\{p, q\} \subseteq G$ implies that there is an $r \in G$ such that $p \cup q \subseteq r$, and (iii) $G \cap D \neq \varnothing$ for every dense open class $D$ of $M$. We remark that $G$ will not be a class of $M$. In the following let $G$ be a fixed collection which is $C$-generic over $M$.

We inductively define a function $\operatorname{den}{ }_{G}()$ whose arguments are elements of $S(R A) \cup T(R A)$. The subscript will be omitted when it is clear from the context. $\operatorname{den}(\mathbf{m})=m$ for each $m \in M$. If $k \in K_{a}$ and $\beta<\theta_{a}$ then $\operatorname{den}(k, \beta)^{\swarrow}=\{(\gamma, i) \mid$ $(\exists p \in G)(\alpha, k, \beta, \gamma, i) \in p\}$. If $k \in K_{\alpha}$ then $\operatorname{den}\left(k^{2}\right)=\left\{\operatorname{den}(k, \beta)^{2} \mid \beta<\theta_{a}\right\}$.

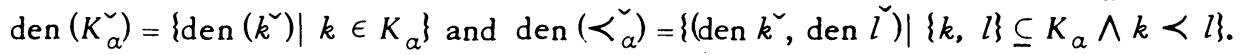

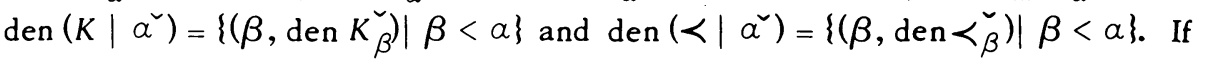
$\{u, v\} \subseteq T(R A)$ then $\operatorname{den}(u \in v)=1$ if $\operatorname{den}(u) \in \operatorname{den}(v)$, and $=0$ otherwise; $\operatorname{den}(u \equiv v)=1$ if $\operatorname{den}(u)=\operatorname{den}(v)$, and $=0$ otherwise. If $\{\phi, \psi\} \subseteq S(R A)$ then $\operatorname{den}(\sim \phi)=1-\operatorname{den}(\phi)$ and $\operatorname{den}(\phi \wedge \psi)=\min \{\operatorname{den}(\phi)$, den $(\psi)\}$. If $(\forall \alpha) \phi \in S(R A)$ then $\operatorname{den}\left(\forall_{a} x\right) \phi=1$ if den $\phi(x / u)=1$ for every $u \in T(R A)$ with $\lambda(u)<\alpha$, and $=0$ otherwise. Finally if $\left(g_{a} x\right) \phi \in T(R A)$ then $\operatorname{den}\left(g_{a} x\right) \phi=\{\operatorname{den}(u) \mid u \in T(R A)$ $\wedge \lambda(u)<\alpha \wedge \operatorname{den} \phi(x / u)=1\}$. Here we have used $\phi(x / u)$ for the result of replacing every free occurrence of $x$ in $\phi$ by $u$. The structure $M[G]$ which we shall henceforth call $N$ is the set $\{\operatorname{den}(u) \mid u \in T(R A)\}$. As we vary the generic $G$ we get various structures $M\left[G^{\prime}\right]$. Note that this definition of den has not been carried out in either $M$ or $N$.

Next we define our forcing language $L(F C)$. The symbols of $L(F C)$ are individual variables $x_{0}, x_{1}, \cdots$, the constants in $T(R A)$, binary predicates $\varepsilon$, $\equiv$, connectives $\Lambda, \sim$, the quantifier $\forall$, a unary functor $K_{(}^{\sim}$, class symbols $M^{\sim}, K^{\smile}$, and a binary predicate $\prec$. The formation rules of $L(F C)$ are those of the predicate calculus. It will be convenient to take $L(F C)$ as a class of $M$. Let $S(F C)$ be the class of sentences of $L(F C)$. For each $\phi \in S(F C)$ we define the notion of satisfaction in $N$ (in symbols $N \vDash \phi$ ) as follows. If $\{u, v\} \subseteq T(R A)$ then $N \vDash$ $u \varepsilon v$ if $\operatorname{den}(u) \in \operatorname{den}(v), N \vDash u \equiv v$ if $\operatorname{den}(u)=\operatorname{den}(v), N \vDash u \varepsilon M^{\sim}$ if $N \vDash u \equiv \mathbf{m}$ for some $m \in M, N \vDash u \equiv K_{v}^{\sim}$ if $\operatorname{den}(u)=\operatorname{den}\left(K_{a}^{\sim}\right)$ for some $a \in O n^{(M)}$ with $N \vDash a$ $\equiv v, N \vDash u \varepsilon K^{\sim}$ if $N \vDash u \varepsilon K_{a}^{\sim}$ for some $\alpha \in O n^{(M)}$, and $N \vDash u \prec^{\sim} v$ if $N \vDash u \equiv k^{\sim}$ and $N \vDash v \equiv l^{\sim}$ for some $\{k, l\} \subseteq K$ with $k \prec l$. Satisfaction is then extended to $S(F C)$ as in ordinary model theory. Note that although $\left.K_{(}\right)$is being used as both an element of $T(R A)$ and as a functor of $L(F C)$, there will be no inconsistency because of our definition of satisfaction. 
If $p$ is a condition and $\phi \in S(F C)$ we say that $p$ forces $\phi$ (in symbols $p \Vdash \phi)$ if $M\left[G^{\prime}\right] \vDash \phi$ for every $C$-generic $G^{\prime}$ over $M$ with $p \in G^{\prime}$. The omitted proofs of Lemmas 1-4 below can essentially be found in [2].

Lemma 1. If $\phi\left(x_{0}, \cdots, x_{n-1}\right)$ is a formula of $L(F C)$ then $\left\{\left(p, u_{0}, \cdots\right.\right.$, $\left.u_{n-1}\right) \mid p \in C \wedge$ eacb $\left.u_{i} \in T(R A) \wedge p \Vdash \phi\left(u_{0}, \cdots, u_{n-1}\right)\right\}$ is a class definable in $M$.

Lemma 2. If $\phi$ is a sentence of $L(F C),\{p, q\} \subseteq C, p \subseteq q$, and $p \Vdash \phi$ then $q \Vdash \phi$.

Lemma 3. If $\phi$ is a sentence of $L(F C)$ then $N \vDash \phi$ if and only if $p \Vdash \phi$ for some $p \in G$.

Lemma 4. If we formulate the axioms of $Z F$ without the axiom of choice in $L(F C)$, i.e., we allow the extra constants, functors and predicates of $L(F C)$ to appear in the axiom of replacement, then $N$ is a model of $Z F$ baving the same ordinals and well-orderable cardinals as $M$.

Next we extend the function occ ( ) to formulas of $L(F C)$. To be precise this should be done inductively, but it comes to the same things if we say that $\operatorname{occ}(\phi)$ is the union of the occ $(u)$ where $u \in T(R A)$ and $u$ occurs in $\phi$. For any $a \in T(R A) \cup L(F C)$ let $\sigma_{0}(a)=\operatorname{occ}(a) \cap\left(K \times O n^{(M)}\right), \bar{\sigma}_{0}(a)=\left\{k \in K\left|\left(\exists \beta \in O n^{(M)}\right)\right|(k, \beta) \epsilon\right.$ $\left.\sigma_{0}(a)\right\}$, and $\sigma_{1}(a)=\bar{\sigma}_{0}(a) \cup(\operatorname{occ}(a) \cap K)$. Here we assume that $K$ is disjoint from $K \times O n^{(M)}$. Define the predicate aut $(\pi)$ if $\pi$ is a finite function $\wedge \delta \pi \cup \rho \pi \subseteq$ $K \wedge \pi$ is $\prec$ order preserving $\wedge x \in \delta \pi \cap K_{a}$ implies $\pi(x) \in K_{a}$. For each $a \epsilon$ $T(R A) \cup L(F C)$ define $\operatorname{acp}(\pi, a)$ if aut $(\pi) \wedge \sigma_{1}(a) \subseteq \delta \pi \wedge \bar{\sigma}_{0}(a)$ is left pointwise fixed by $\pi$. We extend the action of $\pi$ satisfying aut $(\pi)$ first to $u \in T(R A)$ satisfying $\sigma_{1}(u) \subseteq \delta \pi$ and then to $\phi \in L(F C)$ satisfying $\sigma_{1}(\phi) \subseteq \delta \pi$ in the following way. If $x$ is an individual variable then $\pi(x)=x$ and if $m \in M$ then $\pi(\mathbf{m})=\mathbf{m}$.

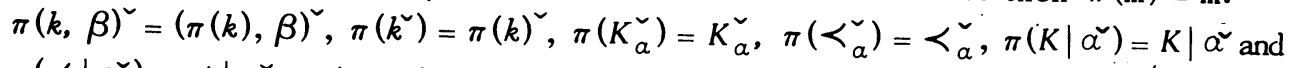
$\pi\left(\langle| \alpha^{\sim}\right)=\langle| \alpha^{\sim} . \pi\left(\begin{array}{lll}u & \varepsilon v\end{array}\right)=\pi(u) \varepsilon \pi(v), \pi(u \equiv v)=\pi(u) \equiv \pi(v), \pi(\phi \wedge \dot{\psi})=$ $\pi(\phi) \wedge \pi(\psi), \pi(\sim \phi)=\sim \pi(\phi), \pi(\forall a x) \phi=\left(\forall_{\alpha} x\right)_{\pi}(\phi)$, and $\pi\left(g_{a} x\right) \phi=\left(g_{a} x\right) \pi(\phi)$. Thus $\pi$ is defined on $L(R A)$. $\pi\left(u \varepsilon M^{\nu}\right)=\pi(u) \varepsilon M^{\sim}, \pi\left(u \varepsilon K^{\sim}\right)=\pi(u) \varepsilon K^{\llcorner}$, $\pi(u \prec v v)=\pi(u) \prec \longleftarrow \pi(v)$, and $\pi\left(u \equiv K_{\nu}^{\smile}\right)=\pi(u) \equiv K_{\pi(v)}^{\sim}$. Connectives are handled as above and $\pi(\forall x) \phi=(\forall x) \pi(\phi)$. Thus $\pi$ is defined on $L(F C)$. There is still one more kind of automorphism that we need for technical reasons. Let $H_{a} \in M$ be the set of all permutations of $\theta_{a}$ which lie in $M$. If $\pi \in H_{a}, k \in K_{a}$ and $a \in T(R A) \cup$ $L(F C)$ we define $\pi^{k}(a)$ in the following way. $\pi^{k}(k, \beta)^{\iota}=(k, \pi(\beta))^{\iota}, \pi^{k}(l, \beta)^{\iota}=$

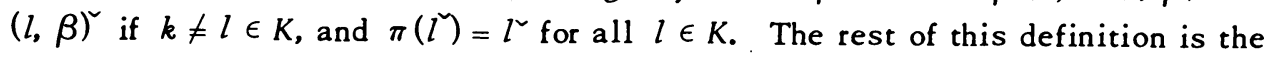
same as in the aut $(\pi)$ case.

If $p \in C$ is a condition let $\sigma_{0}(p)=\{(k, \beta) \mid(\exists \alpha, \gamma, i)(\alpha, k, \beta, \gamma, i) \in p\}$ and let $\bar{\sigma}_{0}(p)=\left\{k \mid(\exists \beta)(k, \beta) \in \sigma_{0}(p)\right\}$. If aut $(\pi)$ and $\bar{\sigma}_{0}(p) \subseteq \delta \pi$ let $\pi(p)=\{(\alpha, \pi(k)$, 
$\beta, \gamma, i) \mid(\alpha, k, \beta, \gamma, i) \in p\}$. If $\pi \in H_{\alpha}, k \in K_{a}$, and $\beta<\theta_{a}$ let $p^{k}=$ $\{(\alpha, k, \beta, \gamma, i) \mid(\alpha, k, \beta, \gamma, i) \in p\}$ and $p^{(k, \beta)}=\{(\alpha, k, \beta, \gamma, i) \mid(\alpha, k, \beta, \gamma, i)$ $\epsilon p\}$. Then let $\pi^{k}(p)=\left(p-p^{k}\right) \cup\left\{(\alpha, k, \pi(\beta), \gamma, i) \mid(\alpha, k, \beta, \gamma, i) \in p^{k}\right\}$. Finally, if $a \subseteq K \times O n^{(M)}$ let $p \mid a=\bigcup\left\{p^{(k, \beta)} \mid(k, \beta) \in a\right\}$. Proofs of Lemmas $5-7$ can be found in [1].

Lemma 5. If $\phi$ is a sentence of $L(F C), \pi \in H_{\alpha}, k \in K_{\alpha}, p \in C$ and $p \Vdash \phi$, then $\pi^{k}(p) \Vdash \pi^{k}(\phi)$.

Lemma 6. If $\phi$ is a sentence of $L(F C), p \in C$ and $p \Vdash t$, then $p \mid \sigma_{0}(\phi) \Vdash \phi$.

Lemma 7. If $\phi$ is a sentence of $L(F C)$, aut $(\pi), p \in C, \bar{\sigma}_{0}(p) \cup \sigma_{1}(\phi) \subseteq \delta \pi$ and $p \Vdash \phi$, then $\pi(p) \Vdash \pi(\phi)$.

For each ordinal $\alpha \in O n^{(M)}$ let $F_{a} \in M$ be the set of all functions $f \in M$ such that $\delta f \subseteq \theta_{a} \wedge \rho f \subseteq\{0,1\} \wedge|f|<\theta_{a}$. The heart of our proof of Theorem 3 consists of the following two perturbation lemmas.

Lemma 8. Let $\alpha(0), \cdots, \alpha(n-1)$ be a finite sequence of ordinals in $O n^{(M)}$ and let $\phi\left(x_{0}, \cdots, x_{n-1}\right)$ be a formula of $L(F C)$ with free variables indicated. For each $i<n$ let $u_{i}=\left(k_{i}, \beta_{i}\right)^{\sim}$ where $k_{i} \in K_{a(i)}, \beta_{i}<\theta_{a(i)},\left(k_{i}, \beta_{i}\right) \notin \sigma_{0}(\phi)$ and the $u_{i}$ are distinct. If $N \vDash \phi\left(u_{0}, \cdots, u_{n-1}\right)$ then there exist $f_{i} \in F_{a(i)}$ such that $f_{i} \subseteq \operatorname{den}\left(u_{i}\right)$ and $N \vDash\left(\forall x_{0}, \cdots, x_{n-1}\right)\left(f_{0} \subseteq x_{0} \varepsilon k_{0}^{\sim} \wedge \cdots \wedge f_{n-1} \subseteq x_{n-1} \varepsilon\right.$ $\left.k_{n-1}^{\sim} \rightarrow \phi\left(x_{0}, \cdots, x_{n-1}\right)\right)$.

Proof. (a) Suppose that $a(i)=\alpha(j)$ but $\left(k_{i}, \beta_{i}\right) \neq\left(k_{j}, \beta_{j}\right)$. Since $\left|p^{\alpha(i)}\right|<$ $\theta_{\alpha(i)}$ for every $p \in C$, there is no $p \in G$ which could force $\left(k_{i}, \beta_{i}\right)^{\swarrow} \equiv\left(k_{j}, \beta_{j}\right)^{\swarrow}$. Thus $\left(k_{i}, \beta_{i}\right)^{\llcorner} \equiv\left(k_{j}, \beta_{j}\right)^{\llcorner}$is false in $N$ and hence we may find a $p \in G$ such that if we let $f_{i}=\left\{(\gamma, j) \mid\left(\alpha(i), k_{i}, \beta_{i}, \gamma, j\right) \in p\right\}$ for $i<n$ then $f_{i}$ and $f_{j}$ will be incompatible.

(b) Suppose that $N \vDash \phi\left(u_{0}, \cdots, u_{n-1}\right)$. Then by Lemma 3 there is a $p \in G$ such that $p \Vdash \phi\left(u_{0}, \cdots, u_{n-1}\right)$. Let $b=\left\{\left(k_{i}, \beta_{i}\right) \mid i<n\right\}$. By Lemma 6 and the definition of $C$-generic we may assume that $\sigma_{0}(p) \subseteq b \cup \sigma_{0}(\phi)$. Let $f_{i}$ be defined from $p$ as in (a). Then by the remark of (a) we can choose $p$ so that $f_{i}$ is incompatible with $f_{j}$ for $i \neq j$, and if $(l, \gamma) \in \sigma_{0}(\phi)$ and $f$ is defined from $(l, \gamma)$ and $p$ as in (a), then $f$ is incompatible with each $f_{i}$ (since $\left.\left(k_{i}, \beta_{i}\right) \notin \sigma_{0}(\phi)\right)$. Clearly $f_{i} \subseteq \operatorname{den}\left(u_{i}\right)$.

(c) Now consider any elements $v_{0}, \cdots, v_{n-1}$ in $T(R A)$ such that $N \vDash \mathbf{f}_{i}$ $\subseteq v_{i} \varepsilon k_{i}$. By the definition of satisfaction in $N$ there exist $\delta_{0}, \cdots, \delta_{n-1}$ in $O n^{(M)}$ such that $w_{i}=\left(k_{i}, \delta_{i}\right)^{2}$ is in $T(R A)$ and $N \vDash v_{i} \equiv w_{i}$ for $i<n$. Our lemma will follow if we can show that $N \vDash \phi\left(w_{0}, \cdots, w_{n-1}\right)$. Take $q \in G$ extending $p$ such that $q \mathbb{f} \mathrm{f}_{i} \subseteq w_{i}$ for $i<n$. We note the assumptions of (b) imply that 
$\left(k_{i}, \delta_{i}\right) \notin \sigma_{0}(\phi)$ for each $i<n$ (because $f_{i}$ and the $f$ of (b) are incompatible functions). Let $d=\left\{\left(k_{i}, \delta_{i}\right) \mid i<n\right\}$. For each $i<n$ choose $\lambda_{i}<\theta_{a(i)}$ such that $\left(k_{i}, \lambda_{i}\right) \notin b \cup d \cup \sigma_{0}(\phi)$ and where the $\lambda_{i}$ are distinct ordinal numbers. Let $\pi_{i} \in H_{a(i)}$ be the transposition $\left(\beta_{i}, \lambda_{i}\right)$, and let $\tau_{i} \in H_{a(i)}$ be the transposition $\left(\lambda_{i}, \delta_{i}\right)$. Let $\pi$ be the composition of the $\pi_{i}^{k_{i}}$, and let $\tau$ be the composition of the $\tau_{i}^{k_{i}}$. Now $q \Vdash f_{i} \subseteq\left(k_{i}, \delta_{i}\right)^{\smile}$ and therefore if $\left(\alpha(i), k_{i}, \beta_{i}, \gamma, j\right) \in p$ then $(\alpha(i)$, $\left.k_{i}, \delta_{i}, \gamma, j\right) \in q$. Hence $\tau \circ \pi(p) \subseteq q$ because $\tau \circ \pi\left(p^{(l, \gamma)}\right)=p^{(l, \gamma)}$ for any $(l, \gamma)$ $\epsilon \sigma_{0}(\phi)$. By inspection $\tau \circ \pi\left(u_{i}\right)=w_{i}$ so by Lemma 5 used $2 n$ times we have $\tau \circ \pi(p) \Vdash \phi\left(w_{0}, \cdots, w_{n-1}\right)$ since we have insured that $\tau \circ \pi(l, \gamma)^{\swarrow}=(l, \gamma)^{\swarrow}$ for each $(l, \gamma) \in \sigma_{0}(\phi)$. By Lemma $2, q \Vdash \phi\left(w_{0}, \cdots, w_{n-1}\right)$. But $q \in G$ and hence by Lemma 3 we get $N \mathbb{t} \phi\left(w_{0}, \cdots, w_{n-1}\right)$. Q.E.D.

Lemma 9. If $\phi$ is a sentence of $L(F C)$, acp $(\pi, \phi)$ and $N \vDash \phi$, then $N \vDash \pi(\phi)$.

Proof. Suppose that $N \vDash \phi$. Then by Lemma 3 there is a $p \in G$ such that $p \Vdash \phi$. By Lemma 6 we may assume that $\sigma_{0}(p) \subseteq \sigma_{0}(\phi)$. Now acp $(\pi, \phi)$ and

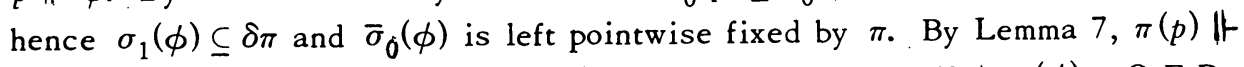
$\pi(\phi)$. But $\pi(p)=p$ so that $p \Vdash \pi(\phi)$. Since $p \in G$ hence $N \Vdash \pi(\phi)$. Q.E.D.

A proof of the following lemma can be found in [1].

Lemma 10. Let $\phi(x)$ be a formula of $L(F C)$ with free variables indicated. If $N \vDash(\exists ! x) \phi(x)$, then there is a $u \in T(R A)$ with occ $(u) \subseteq$ occ $(\phi)$ such that $N \vDash \phi(u)$.

Lemma 11. If $\{u, v\} \subseteq T(R A)$ and $N \vDash u \equiv v$, then there is a $w \in T(R A)$ with $\sigma_{i}(w) \subseteq \sigma_{i}(u) \cap \sigma_{i}(v)$ for $i<2$ sucb that $N \vDash u \equiv w$.

Proof. Let $(k, \beta) \in \sigma_{0}(u)-\sigma_{0}(v)$ where $k \in K_{a}$ and let $u(y)$ be the result of replacing $(k, \beta)^{\smile}$ in $u$ by the variable $y$. Now $(\forall x)(x \in u(y)$ iff $x \varepsilon v)$ can be thought of as a formula of $L(F C)$ which is satisfied in $N$ by $\operatorname{den}(k, \beta)^{\swarrow}$. By Lemma 8 there is an $f \in F_{a}$ such that $N \vDash(\forall y)\left(f \subseteq y \varepsilon k^{2} \rightarrow(\forall x)(x \varepsilon u(y)\right.$ iff $x \quad \varepsilon v)$ ). Then by Lemma 10 there is a $w \in T(R A)$ such that $N \vDash u \equiv w$ and $\operatorname{occ}(w) \subseteq \operatorname{occ}(u)-\{(k, \beta)\} \cup\{k\}$. Next let us suppose that $k \in \sigma_{1}(u)-\sigma_{1}(\nu)$, $k \notin \bar{\sigma}_{0}(u) \cup \bar{\sigma}_{0}(v)$ where $k \in K_{\alpha}$. Choose a $\pi$ such that aut $(\pi), \delta \pi=\sigma_{1}(u) \cup$ $\sigma_{1}(v)$ where $k$ is the only element of $\delta \pi$ which is moved by $\pi$. If we apply Lemma 9 to $N \vDash u \equiv v$, we get $N \vDash \pi(u) \equiv v$ and hence $N \vDash \pi(u) \equiv u$. Let $k_{0}$ $\left(k_{1}\right)$ be the greatest (least) element in $\sigma_{1}(u) \cap K_{a}$ which is below (above) $k$ in the $\prec$ ordering, and let $u(y)$ be the result of replacing $k^{\swarrow}$ in $u$ by the variable $y$. By another application of Lemma 9 we can show that $N \vDash \pi^{\prime}(u) \equiv u$ where $\pi^{\prime}$ is fixed on $\sigma_{1}(u)-\{k\}$ and moves $k$ into any element of $\left\{x \in K_{\alpha} \mid k_{0}<x<k_{1}\right\}$.

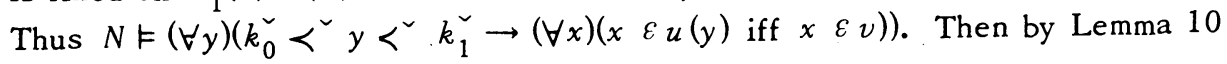


there is a $w \in T(R A)$ such that $N F u \equiv w$ and $\operatorname{occ}(w) \subseteq \operatorname{occ}(u)-\{k\}$. Our lemma follows by repeated use of the two preceding elimination methods. Q.E.D.

Next we define another ramified language $L^{*}(R A)$. This time the definition will occur in $N$ using the language $L(F C)$ without individual constants. In general the formation rules of $L^{*}(R A)$ are the same as those of $L(R A)$. The only difference is in the introduction of individual constants. These constants consist of an $\mathrm{m}$ for each $m \in M$, for each $a \in O n^{(M)}$ constants $K_{a}^{*}, \prec_{a}^{*}, K\left|\alpha^{*}, \prec\right| \alpha^{*}$, for each $k \in \operatorname{den}\left(K_{a}^{\sim}\right)$ a constant $k^{*}$, and for each $s \in k$ a constant $s^{*}$. In the above process the mapping $x \leftrightarrow x^{*}$ is definable in $N$. We define a function occ ${ }^{*}$ by $\operatorname{occ}^{*}\left(k^{*}\right)=\{k\}$, occ ${ }^{*}\left(s^{*}\right)=\{s\}$, the remaining clauses of this definition are obvious. We similarly define $\lambda^{*}$. The more interesting case is when we come to $\operatorname{den}^{*} \cdot \operatorname{den}^{*}\left(K_{a}^{*}\right)=\operatorname{den}\left(K_{a}^{\sim}\right), \operatorname{den}{ }^{*}\left(\prec_{a}^{*}\right)=\operatorname{den}\left(\prec_{a}^{\sim}\right), \operatorname{den}^{*}\left(K \mid \alpha^{*}\right)=\operatorname{den}\left(K \mid \alpha^{\swarrow}\right)$, $\operatorname{den}^{*}\left(\prec \mid a^{*}\right)=\operatorname{den}\left(\prec \mid \alpha^{<}\right), \operatorname{den}^{*}\left(k^{*}\right)=k$, and $\operatorname{den}^{*}\left(s^{*}\right)=s$. The rest of this definition is the same as that of den. Continuing in this vein we can also define a language $L^{*}(F C)$, occ ( ) applied to members of $L^{*}(F C)$, and for $a \in T^{*}(R A) \cup$ $L^{*}(F C)$ the notions $\sigma_{0}^{*}(), \bar{\sigma}_{0}^{*}()$, and $\sigma_{1}^{*}()$. aut ${ }^{*}()$ and $\operatorname{acp}^{*}()$ then have their obvious definitions. It should be clear that the starred notions can all be defined in $N$ using formulas of $L(F C)$ without individual constants.

Lemma 12. There is a one-one mapping $u \leftrightarrow u^{*}, \phi \leftrightarrow \phi^{*}$ for $u \in T(R A), u^{*}$ $\in T^{*}(R A), \phi \in S(F C), \phi^{*} \in S^{*}(F C)$ such that $\operatorname{den}(u)=\operatorname{den}{ }^{*}\left(u^{*}\right)$ and $N \vDash \phi$ iff $N \vDash \phi^{*}$; consequently we bave starred versions of Lemmas 8 through 11 .

Lemma 13. The following sentences can be formulated in $L(F C)$ without individual constants and are true in $N$.

(i) $(\forall x)(\exists y)\left(y \in T^{*}(R A) \wedge x=\operatorname{den} *(y)\right)$.

(ii) $(\forall x)(\exists y)\left(x=\operatorname{den}^{*}(y) \wedge(\forall z)\left(x=\operatorname{den}^{*}(z) \rightarrow \sigma_{0}^{*}(y) \subseteq \sigma_{0}^{*}(z) \wedge \sigma_{1}^{*}(y) \subseteq \sigma_{1}^{*}(z)\right)\right)$.

Proof of Theorem 3. For any $x \in N$ and $i<2$ we let $s_{i}(x)=\bigcap\left\{\sigma_{i}^{*}(u) \mid u \epsilon\right.$ $\left.T^{*}(R A) \wedge x=\operatorname{den}^{*}(u)\right\} . \bar{s}_{0}(x)$ is then defined as in (5). The $s_{i}()$ are definable in $N$ by Lemma 13. Next define an ordering $R \subseteq X^{2} N$ as follows. If $x, y \in N$ put $(x, y) \in R$ if in the lexicographic ordering determined by den $(\prec)$ and first differences on $P(\alpha)$, either (i) $s_{0}(x)$ precedes $s_{0}(y)$, or $(\mathrm{ii}) s_{0}(x)=s_{0}(y)$ and $s_{1}(x)$ precedes $s_{1}(y)$, or (iii) $s_{0}(x)=s_{0}(y)$ and $s_{1}(x)=s_{1}(y)$ and there is a $u \in T^{*}(R A)$ such that $x=\operatorname{den}^{*}(u), s_{i}(x)=\sigma_{i}^{*}(u)$ for $i<2$ and for any $v \in T^{*}(R A)$ if $y=$ $\operatorname{den}^{*}(v)$ and $\sigma_{i}^{*}(u)=\sigma_{i}^{*}(v)$, then $u$ precedes $v$ in some canonical well ordering of $\left\{v \in T^{*}(R A) \mid s_{i}(x)=\sigma_{i}^{*}(v)\right.$ for $\left.i<2\right\}$. It is clear from this definition that $\operatorname{den}\left(\prec^{\sim}\right) \subseteq R$. aut ( ) will be interpreted in $N$ as the predicate aut ${ }^{*}()$. If aut $(\pi)$ and $x \in N$ let $\operatorname{acp}(\pi, x)$ if $s_{1}(x) \subseteq \delta \pi$ and $\bar{s}_{0}(x)$ is left pointwise fixed by $\pi$. If $u \in T^{*}(R A), x=\operatorname{den}^{*}(u)$, and $s_{i}(x)=\sigma_{i}^{*}(u)$ for $i<2$ we put $\pi(x)=\operatorname{den}^{*}(\pi u)$. By 
Lemma 9 the latter is well defined. Most of the axioms (1)-(15) will follow immediately from these definitions and Lemmas (1)-(13). We will only discuss those where some computation is necessary.

Re (6). Let $k \in K_{\alpha}$ and $\beta<\theta_{a}$. If there were a $u \in T(R A)$ such that $N \vDash$ $(k, \beta)^{\sim} \equiv u$ and $\sigma_{0}(u)=\varnothing$ then by Lemma 8 there would be an $f \in F_{a}$ such that $N \vDash(\forall x)\left(f \subseteq x \in k^{2} \rightarrow x \equiv u\right)$. Let $f \subseteq g \in F_{a}$ where $g$ is incompatible with $\operatorname{den}(k, \beta)^{\swarrow}$. But then $N \vDash(\exists x)\left(\mathrm{g} \subseteq x \varepsilon k^{\swarrow}\right)$ since no $p \in C$ could possibly force its negation. Thus there is a $\gamma<\theta_{a}$ with $\beta \neq \gamma$ such that $N \vDash(k, \beta)^{\smile} \equiv(k, \gamma)^{\smile}$, a contradiction because this could not possibly be forced. By Lemma 12 this implies that if $x=\operatorname{den}(k, \beta)^{\smile}$ then $s_{0}(x)=\{x\}$. A similar result holds for $k^{\swarrow}$ using Lemma 9 instead of Lemma 8.

$\operatorname{Re}(7)$. That $s_{j}(y) \subseteq \bigcup\left\{s_{j}(x) \mid x \in y\right\}$ easily follows from (9), the latter an immediate consequence of Lemma 12. For the converse inclusion note that if $y$ is finite then the restriction of $R$ to $y$ is a well ordering and hence any $x \in y$ is definable from $y, R$, and some integer $n \in \omega$.

Re (12). Assuming the hypotheses of (12) it is clear from (15) that $x \in y$ implies $\pi(x) \in \pi(y)$. Suppose that $s_{1}(y)=a$. Then $u=\left(g_{a} x\right) \bigvee_{k} \epsilon_{a} x \equiv k^{*}$ belongs to $T^{*}(R A), \operatorname{den}^{*}(u)=a$, and by the methods of (6) we can show that $s_{i}(a)=\sigma_{i}^{*}(u)$. Thus if acp $(\pi, y)$ then $\operatorname{acp}(\pi, a)$ and it easily follows from the form of $u$ that $\pi(a)=\{\pi(x) \mid x \in a\}$. Now $(\forall x)\left(x \in y \rightarrow s_{1}(x) \subseteq a\right)$ and hence by Lemma $9(\forall x)\left(x \in \pi(y) \rightarrow s_{1}(x) \subseteq \pi(a)\right)$. In this way we can show that acp $\left(\pi^{-1}, x\right)$ for all $x \in \pi(y) \cup\{\pi(y)\}$. Now let $z \in \pi(y)$. Then $\pi^{-1}(z) \in \pi^{-1}(\pi(y))=y$ by (13), (14) and $\pi\left(\pi^{-1}(z)\right)=\left(\pi \pi^{-1}\right)(z)=z$. So every member of $\pi(y)$ is of the form $\pi(x)$ for some $x \in y$, i.e., $\pi(y) \subseteq\{\pi(x) \mid x \in y\}$. Q.E.D.

\section{REFERENCES}

1. P. J. Cohen, Set theory and the continuum hypothesis, Benjamin, New York, 1966. MR 38 \# 999.

2. W. B. Easton, Powers of regular cardinals, Ann. Math. Logic 1 (1970), 139-178. MR $42 \# 4392$.

3. E. Ellentuck, The universal properties of Dedekind finite cardinals, Ann. of Math. (2) 82 (1965), 225-248. MR 31 \#429.

4. - The first order properties of Dedekind finite integers, Fund. Math. 63 (1968), 7-25. MR 39 \#53.

5. - Extension methods in cardinal arithmetic, Trans. Amer. Math. Soc. 149 (1970), 307-325. MR $41 \# 1524$.

6. - Almost combinatorial Skolem functions, J. Symbolic Logic 35 (1970), 6572. MR $43 \# 7316$.

7. L. Hay, The co-simple isols, Ann. of Math. (2) 83 (1966), 231-256. MR 34 \# 5671.

8. A. Mostowski, Formal system of analysis based on an infinitistic rule of proof, Proc. Sympos. Foundations of Math., Infinitistic Methods (Warsaw, 1959), Pergamon, Oxford; PWN, Warsaw, 1961, pp. 141-166. MR $36 \# 3631$. 
9. A. Nerode, Extensions to isols, Ann. of Math. (2) 73 (1961), 362-403. MR 24 \# A1215.

10. -..., Arithmetically isolated sets and nonstandard models, Proc. Sympos. Pure Math., vol. 5, Amer. Math. Soc., Providence, R.I., 1962, 105-116. MR 26 \# 1251.

DEPARTMENT OF MATHEMATICS, RUTGERS UNIVERSITY, NEW BRUNSWICK, NEW JERSEY 08903

DEPARTMENT OF MATHEMATICS, INSTITUTE FOR ADVANCED STUDY, PRINCETON, NEW JERSEY 08540 